\title{
Experimental study on drop breakup time and breakup rate with drop swarm in a stirred tank
}

\author{
Han Zhou ${ }^{1}$, Xiong $\mathrm{Yu}^{2}$, Bo Wang ${ }^{1}$, Shan Jing ${ }^{1}$, Wenjie $\mathrm{Lan}^{3}$, and Shaowei $\mathrm{Li}^{4}$ \\ ${ }^{1}$ Tsinghua University \\ ${ }^{2}$ Tsinghua University Institute of Nuclear and New Energy Technology \\ ${ }^{3}$ China University of Petroleum (Beijing) \\ ${ }^{4}$ Institute of Nuclear and New Energy Technology
}

April 28, 2020

\begin{abstract}
Drop breakup experiments were carried out in a stirred tank using the high-speed online camera. Breakup behaviors of drop breakup time, multiple breakage, and breakup rate were investigated. Experimental results show that the drop breakup time is mainly controlled by the interfacial tension and drop diameter, while is almost independent of the rotating speed. Besides, the dispersed phase viscosity has a slight influence on the breakup time. An empirical correlation for the breakup time is proposed and is further verified by comparing with the results of Solsvik and Jakobsen (Chem. Eng. Sci., 2015, 131: 219-234). The percentage of multiple breakage comparing to binary breakup was statistically counted. The results indicated that the dimensionless drop diameter $\eta=d / d_{\max }$ can be adopted to characterize the proportion of binary breakup. Finally, the breakup rate was experimentally measured and the breakup probability was calculated using the inverse method.
\end{abstract}

\section{Introduction}

Turbulent liquid-liquid dispersion is of critical important in areas of chemical engineering, such as solvent extraction $^{1,2}$, chemical reaction ${ }^{3}$, and emulsion process ${ }^{4-6}$, etc. One of the key parameters in those systems is the dispersed phase size distribution, as it determines the contact area between two phases and thus controls the mass, momentum, and heat transfer rate. In the turbulent regime, the evolution of the drop size distribution (DSD) is caused by behaviors of drop breakage and coalescence. ${ }^{7-11}$ For the case where the volume fraction of the dispersed phased is very low, the influence of the drop coalescence on the DSD evolution can be omitted compared to the drop breakage. Thus, the time and space distribution of the particle size can be characterized by quantifying the drop breakup behavior. To achieve the goal above, it is necessary to carry out in-depth and detailed experimental and theoretical researches on the mechanism of the drop breakup.

In earlier experimental studies, the drop breakup data was usually reversely deduced from statistical analysis of the droplet size distribution. ${ }^{12-16}$ Correspondingly, researchers established various breakup models based on mathematical and mechanical analysis. ${ }^{17-23}$ This analytical method is still adopted by many researchers over the years. Conclusions based on this method might be well applied to specific devices and systems, but often have unverified extensibility and accuracy when applied to other application conditions. To understand dynamics of the drop breakup, the first job is to obtain the direct experimental data of drop breakup. Over the years, the development of high-speed camera technology has provided strong support for the observation of drop breakup behavior. Employing high-speed camera equipment, researchers 
carried out a series of experiments to explore the mechanism of drop breakup. Those works were performed with various systems, operating conditions, and different experimental facilities. The topic mainly focused on the following aspects: breakup possibility ${ }^{24-31}$, number of fragments ${ }^{24,25,28,32-37}$ and daughter drop size distribution ${ }^{24-26,28,29,33,35,38-41}$, etc. Despite much efforts that have been done to investigate the drop breakup, it is still a long way to adequately understand breakup phenomena due to the complexity of breakup dynamics in turbulent liquid-liquid dispersions. At present, the direct experimental study on drop breakup behavior, especially the study on breakup time and breakup rate which will be briefly reviewed in the next section, is still very limited.

Therefore, the drop breakup time and breakup rate were experimentally quantified in the present work. A series of experiments were designed to systematically investigate the influences of physical properties and operating conditions, and furtherly, corresponding mathematical models were built to predict the drop breakup time and breakup rate. The goal of this study is to obtain an in-depth understanding of drop breakup processes and to provide direct experimental data for the construction and verification of breakup models.

The study is organized as follows. In the next section, a brief review of the experimental investigation on drop breakup time and breakup rate is present. Whereafter, the experimental equipment and research methods adopted in this study are introduced in detail. External turbulence parameters affecting drop breakup are estimated. The experimental results for the breakup time, breakup rate and the problems of multiple fragmentations are discussed in the results and discussion section. Moreover, the modeling ideas of breakup time existing in the literature are summarized and analyzed in this section. Finally, the conclusion is present in the last section.

\section{Review of experimental study on drop breakup time and breakup rate}

In this study, we mainly focus on the breakup time and breakup rate generated upon drop breakup. Corresponding researches in the literature were reviewed in this section.

The drop breakup time is an important parameter to understand the mechanism of drop breakup. At present, the parameter was obtained mainly by single drop experiments. Heskech et al. ${ }^{42}$ measured a limited number of drop breakup events and obtained the breakup time of silicone oil in a horizontal pipeline. The results showed that the breakup time was 19-59 ms for the mother drop with size 3.40-4.55 mm. Eastwood et al. ${ }^{16}$ found that the elongated drop can break up owing to capillary effects. Meanwhile, they pointed out that the viscosity of the dispersed phase is an important parameter affecting the breakup time. Andersson and Andersson ${ }^{43}$ carried out single drop breakup experiments in turbulent flows. They compared the breakup time of drops with the turbulent time scales and pointed out that only large turbulent eddies are effective in drop breakup ${ }^{43}$. Maaß and Kraume ${ }^{30}$ investigated the drop breakup time in a rectangular channel where a single blade was fixed internally. Their statistical criterion of breakup time is the duration of the whole process from the drop entering the flow field to the final breakup. It should be noted that this time often includes circulation time, where the drop may remain subspherical. On the premise above, they found that the breakup time is not a const but approximately obey a $\beta$-distribution for the drop of a certain size. Furthermore, they discussed the breakup time based on the peak values of the $\beta$-distribution. Experimental data showed that the breakup time monotonically increases with increasing of the drop diameter and decreases with increasing of the energy dissipation rate. Solsvik and Jakobsen ${ }^{34}$ measured the breakup time by single drop breakup experiments in a stirred tank. They scaled the breakup time as the duration taken from the initial deformation of a spherical mother drop to the terminus where the fragments were totally generated. They further defined the breakup process as the breakup cascade ${ }^{37}$. According to their study, the breakup time holds a significant dependence on the drop diameter and increases monotonously with the increase of drop size. Ashar et al. ${ }^{36}$ measured the time for droplet deformation in a rotor-stator mixer. They found out that 
the breakup time is positively scaled with the weber number. Meanwhile, they point out that turbulent eddies with the size close to the target drop control the breakup process.

Despite researchers provided important experimental data of drop breakup time through single drop experiments, the quantitative laws of how physical properties and operating parameters influence the breakup time are still limited. Moreover, the sufficiently predictable model for drop breakup time is also in lack.

Recently, experiments have been carried out by Li's group to measure the breakup rate directly in a pulsed disc and doughnut column ${ }^{38-40,44}$ and in a pump-mixer ${ }^{41,45}$. In the above study, the multiple breakup process is treated mathematically as a sequence of the binary breakup. In this study, experimental data of the breakup rate are also provided. Moreover, we make a systematic analysis of the process of multiple breakage and probe into the influence rules of different factors on multiple breakup.

\section{Drop breakage experiments}

\section{Experimental}

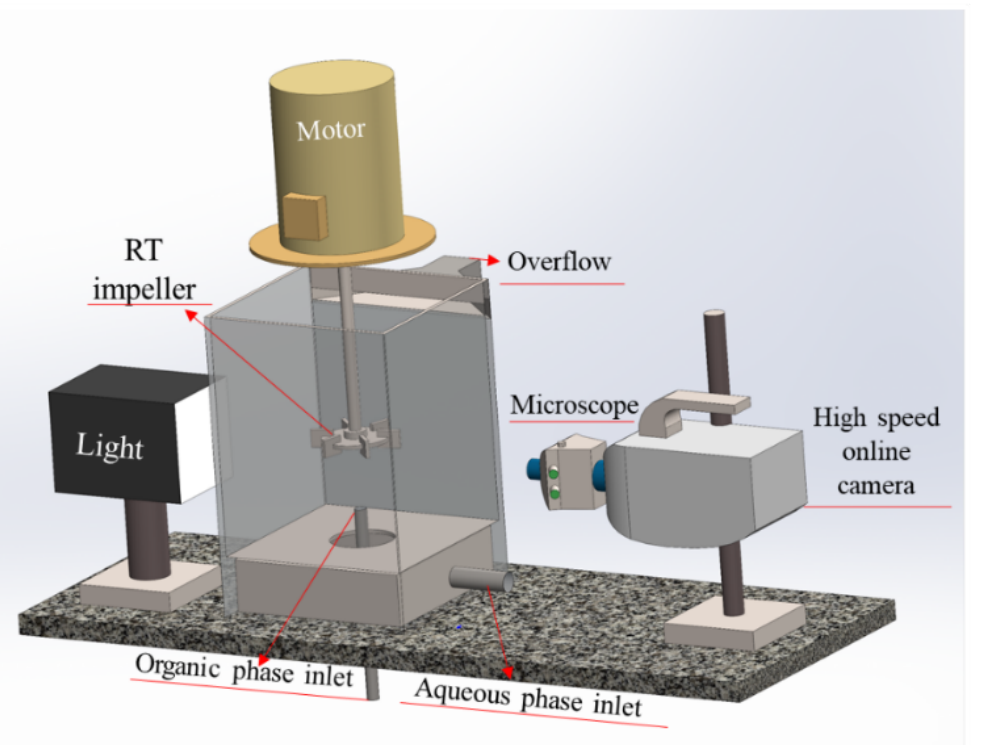

Figure 1 Schematic diagram of the experimental apparatus

The drop breakup experiments under drop swarm were carried out in a stirred tank. The Rushton Turbine (RT) impeller was used to disperse the immiscible two phases. The schematic diagram of the apparatus is shown in Figure 1. Key parameters of the setup are presented in Table 1. A high-speed online camera (Olympus i-Speed TR) connecting with a microscope (Olympus SZ61) was used to observe and record drop breakup processes. The recording rate was fixed as 5000 frames per second and the magnification of the microscope was adjusted between 0.67 and 1.2, enabling the resolution of the captured image of 0.010-0.018 $\mathrm{mm} /$ pixel. In this work, the diameter of the broken droplet is typically larger than $0.2 \mathrm{~mm}$, which means that the settings above can adequately capture the droplet profile.

Table 1 Key parameters of the stirred tank

\begin{tabular}{lll}
\hline & Dimensions $(\mathrm{mm})$ & Details \\
\hline Impeller diameter & 44 & Containing six blades with $8.8 \mathrm{~mm}$ high and $2 \mathrm{~mm}$ thick
\end{tabular}




\begin{tabular}{lll}
\hline & Dimensions $(\mathrm{mm})$ & Details \\
\hline Stirred tank & $100 \times 100 \times 100$ & Refers to the available liquid volume in the stirred tank \\
Mounting height of the impeller & 50 & The distance between the tank bottom and the impeller \\
\hline
\end{tabular}

In this study, the continuous phase is deionized water and five sets of organic liquids are used as the dispersed phase to investigate the incluence of the interfacial tension and dispersed phase viscosity. The physical properties of the dispersed phased are presented in Table 2.

\section{Table 2 Physical Properties of the Dispersed-Phase $\left(25^{\circ} \mathrm{C}\right)$}

\begin{tabular}{lllll}
\hline System No. & Dispersed-phase & $\rho_{\delta}(\mathrm{kg} / \mathrm{m} 3)$ & $\mu_{\delta}(\mathrm{mPa} \cdot \mathrm{s})$ & $\sigma(\mathrm{mN} / \mathrm{m})$ \\
\hline 1 & n-dodecane & 750.1 & 1.64 & 34.61 \\
2 & 2vol\%TBP/ n-dodecane & 754 & 1.64 & 19.58 \\
3 & 10vol\%TBP/n-dodecane & 778 & 1.65 & 11.67 \\
4 & silicone oil_1 & 937 & 9.82 & 25.35 \\
5 & silicone oil_2 & 962 & 86.0 & 31.28 \\
\hline
\end{tabular}

To begin the experiment, the stirred tank was firstly filled with the deionized water, then the motor was started to turn on the impeller. The rotating speed of the impeller was controlled by adjusting the frequency of the motor. After steady state was achieved, the organic phase was injected into the stirred tank from the bottom. Then videos with a timer-period of $1.334 \mathrm{~s}$ were recorded. The system was cleaned and restarted every 30 seconds until enough videos were obtained. In this study, we recorded 50 videos for each experimental condition. It should be noted that the volume fraction of the dispersed phase is no more than $2 \%$ for each operating condition, thus the coalescence between drops can be omitted and only the drop breakup behavior is considered. The breakup event was manually tracked in the video, and the duration of the breakup process, i.e. the breakup time, and the number of fragments were recorded. The breakup rate was also measured using Equation 1, which is consistent with the method adpoted by our previous study ${ }^{45}$.

Where $\Gamma(d)$ is the breakup rate of the drop with a diameter of $d . t_{c}$ is the time duration of the video. $n$ $(d)[?] d$ denotes the number density of the droplets with diameter in the range of [?] $d$ about $d$, while $n_{b}(d$ )[?] drepresents the number density of the broken drops.

\section{Calculation of the disruptive stress}

The rotating speed of impeller was larger than $330 \mathrm{rpm}$ in this study, corresponding to the impeller Reynolds number Re larger than 10000 . Zhang et al. ${ }^{46}$ indicated that the velocity fluctuation levels show Reynolds independent behavior for Reynolds numbers equal to or higher than 6000. Thus, the local dissipation rate of the turbulent eddies can be modeled using Equation 2.

Where $k$ is the turbulent kinetic energy, which is roughly estimated using in a cylindrical vessel with four equispaced baffles. ${ }^{47,48} \Lambda$ donates the distance over which the vortex velocity varies significantly, and is approximately $0.14 D .47,48$ Therefore, the turbulent eddy dissipation rate in the turbine impeller discharge flow is estimated as:

Where $N$ is the rotating speed of the impeller and $D$ is the diameter of the impeller. Equation 3 was also adopted by Tsouris and Tavlarides ${ }^{22}$ and Han et al. ${ }^{49}$. In this study, the experimental equipment is a cubic stirred tank without baffles, the power consumption is about $75 \%$ of that of the cylindrical vessel with four equispaced baffles under the same rotating speed. ${ }^{50}$ Corresponding, the turbulence eddy dissipation should also be reduced by $25 \%$, resulting: 
In this study, the largest length scale of the turbulent eddies is of the order of the impeller radius ${ }^{22}$, i.e. . The minimum size of eddies in the inertia subrange can be calculated according to the Kolmogorov microscale, i.e., . In this study, the upper limit of the is approximately $3 \mathrm{e}-5 \mathrm{~m}$ which is much smaller than the diameter of the broken drops. Thus, the breakage is thought to be caused by the turbulent eddies lying in the inertial subrange. In this case, the velocity difference between any two points with a distance of can be calculated using Equation 5. ${ }^{51,52}$ Thus, the disruptive stress acting on a drop of diameter $d$ is obtained using Equation $6 .^{53}$

Where $\beta=2.0$ according to Luo et al. ${ }^{23}$

\section{Results and discussion}

\section{Experimental breakup time}

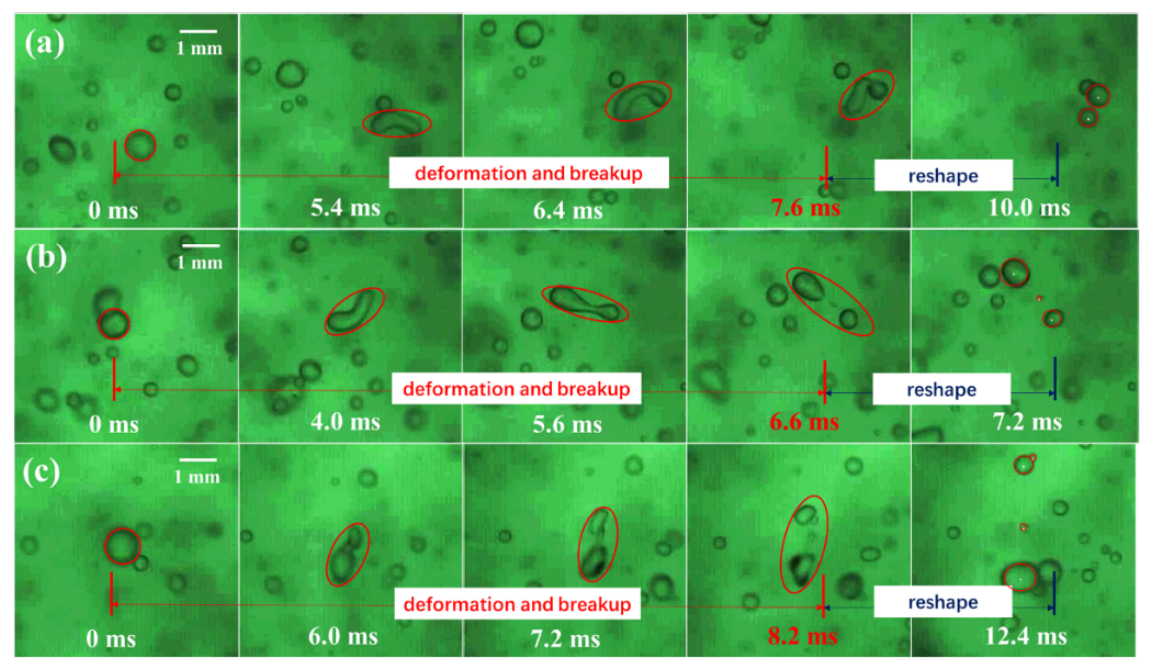

Figure 2 Broken sequences of drops and corresponding breakup times, $N=390 \mathrm{rpm}, \mathrm{n}$-dodecane. (a) binary breakup; (b) ternary breakup; (c) quaternary breakup.

The breakup of a drop is a complicated process in the turbulent flow. At present, the quantitative description of the breakup time is not well addressed in literature as the breakup definition is conflicting in various studies $^{37}$. In this work, we adopted a quantitative method, that is, recording the time since the spherical droplet before deformation to the moment when the last daughter droplet is formed. The duration of the whole process is the breakup time ${ }^{54}$. Generally, the drop undergoes the deformation, stretch or revolve until generating fragments, as is shown in Figure 2. It is indicated that fragments experience the reshaping process after the breakage. The drop breakup time characterizes how fast the drop breaks up under the external disruptive stress. Considering that the reshaping process is controlled by the interfacial tension of the drop, the drop will spontaneously reform into a sphere even if the external forces are withdrawn. Therefore, the duration of the reshaping process is not included in the statistics of breakup time. The breakup time in this study is thus equivalent to the time of deformation and breakup, as shown in Figure 2. In the subsequent analysis, the influences of the fragment number, size distribution of the fragment, rotating speed, interfacial tension and dispersed phase viscosity on the breakup time were discussed.

As is indicated in Figure 2, before breaking up, the drop deformed with a different magnitude due to the turbulent velocity fluctuation. The deformation process is affected by various factors, such as deformation position, instantaneous fluctuation velocity, and direction, droplet trajectory, etc. This leads to certain 
randomness in the process of a drop breaking up. Corresponding, the breakup time will not be constant for a drop with a certain diameter. Experimental data indicated that the measured breakup time has a large variance. ${ }^{30,34}$ Such phenomena were also observed in this study. For detail, the distributions of the breakage time were analyzed using the index of the relative deviation $\left(d_{r}\right)$, i.e., , as shown in Figure 3 . Figure 3 exhibits the influence of the rotating speed, interfacial tension and the dispersed phase viscosity on the distribution of the relative deviation. The distributions show the similar distribution for different experimental conditions. Moreover, the distribution is approximately symmetric over $d_{r}=0$, which indicate symmetric frequency distribution of the breakup time around the arithmetic average value $t_{b, a v e}$. Accordingly, the arithmetic average value $t_{b, a v e}$ can be reasonably adopted for the subsequent analysis of the breakup time. As will be discussed in the following sections, $t_{b, a v e}$ is affected by droplet size, rotating speed of the mixer, as well as physical properties of the liquids.
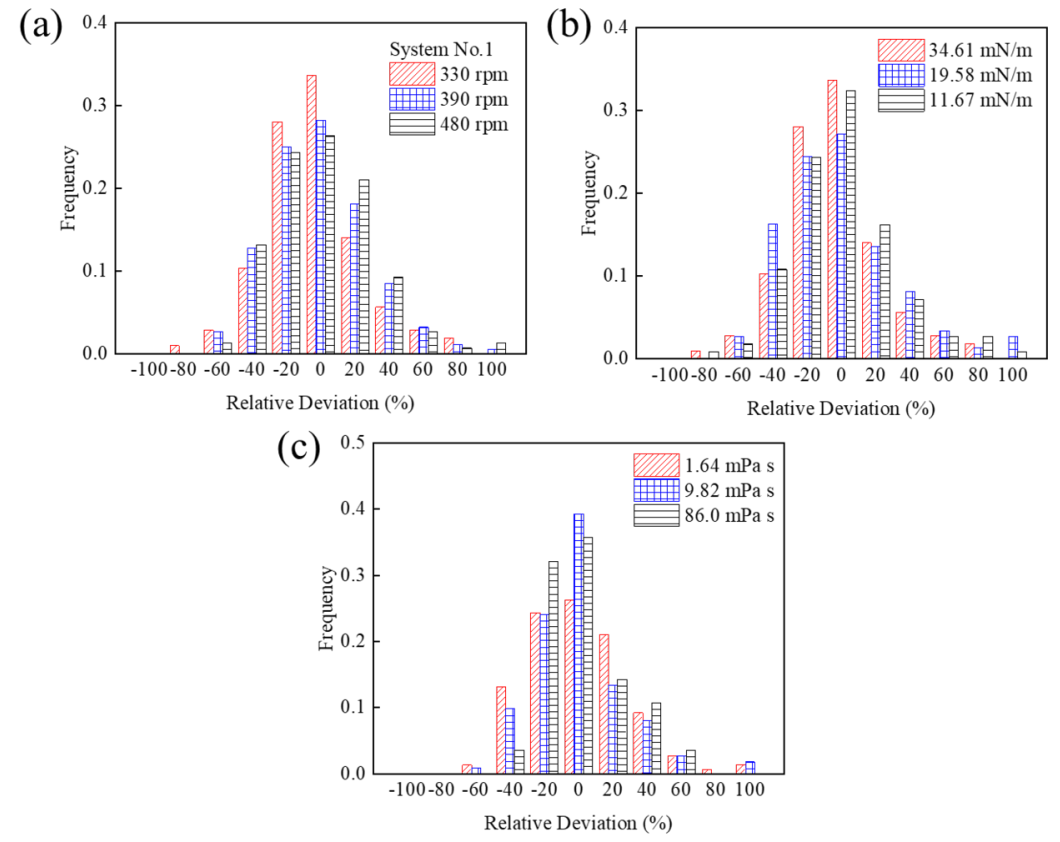

Figure 3 Relative deviation plots of the experimental breakup time. (a) System No.1, N=330 $480 \mathrm{rpm}$; (b) System No.1-3, $N=330 \mathrm{rpm}$; (c) System No.1,4-5, $N=480 \mathrm{rpm}$.

The size distribution of the daughter drop (DDSD) is the product of different drop breakup processes. Recently, several studies were carried out to investigate the influence factors on the DDSD. ${ }^{28,35,41,44}$ However, whether the volume fraction of the fragment corresponds to different drop breakup time is still a problem to be settled. In this work, the influence of the daughter droplet size on the breakup time is analyzed through binary breakup events. By dividing the volume fraction of the smaller fragment $\left(f_{v}\right)$ into several intervals, the arithmetic average breakup time of the binary breakup is determined and is presented in Figure 4. Figure 4a-c show the results under different rotating speeds. It can be seen that the breakup time is slightly higher for the higher $f_{v}$, and the phenomenon is relatively obvious for the lower rotating speed. For the process of drop deformation, the energy is transferred from the surrounding turbulent eddies to the drop surface. An increase in $f_{v}$ usually characterizes the greater surface area changes before and after drop deformation, corresponding to the greater energy requirement from surroundings. With the rough assumption that the energy transfer rate at the drop surface is constant and not affected by other conditions, the larger energy demand thus means longer transfer time, which eventually leads to the increase of breakup time. However, the above discussion is based on a very subjective hypothesis. Practically, due to the complexity of the drop 
breakup process and energy transfer in the turbulent field, the breakup time and DDSD are more likely to be determined by the trajectory of drop deformation. As the trajectory is complex and is influenced by multiple factors, the relationship between the DDSD and the breakup time is often inconspicuous, especially for the stronger external flow field and the smaller interfacial tension, as is shown in Figure 4c-g. Reasonably, the influence of the daughter droplet volume fraction on the breakup time is no longer considered in the following discussion.

Figure 5 shows the influence of the fragment number on the drop breakup for systems No.1-5. It is indicated that the breakup time is almost impervious to the fragment number. For multiple breakages, the drop generally breaks up into two main larger drops and several concomitant satellite droplets. The formation of the satellite droplet exists in the final phase from deformation to breakage, as is shown in Figure $2 \mathrm{~b}-\mathrm{c}$. The time for the satellite to be generated can be neglected compared with the deforming process. The dominant influencing factor of the breakup time is the drop deforming trajectory. In this case, the breakup time is arguably independent of the number of fragments. Meanwhile, it should be pointed out that the drop may also break up into several fragments of similar size, leading to a discrepant breakup time compared to the binary breakup. However, the occurrence probability of this breakup mode is very small in this study and is not enough to have a significant impact on the average breakup time. Therefore, it is still reasonable to consider that the influence of the fragment number on the breakup time can be ignored in this work.

Based on the discussion above, the average breakup time was measured for mother droplet with a certain size ignoring the effect of the number or size distribution of the daughter droplets. Figure 6a shows the average breakup time at different rotating speed. It indicates that the rotating speed has an inapparent influence on the average breakup time. This means that the energy transfer rate at the drop surface is little changed with different rotating speeds in this study. Figure $6 \mathrm{~b}$ shows the influence of the interfacial tension on the average breakup time. The results reveal that the lower interfacial tension will result in a longer breakup time. Solsvik and Jakobsen observed similar behavior in comparing the breakup time of different experiment systems ${ }^{34}$. They attributed the higher breakup time for drops with lower interfacial tension to the relatively higher viscous grade, which can result in a larger degree deformation before drop breakup $^{34}$. This explanation can make sense as similar breakup behavior was observed for the drops with higher viscosity and lower interfacial tension. However, our experimental results showed that the breakup time is more sensitive to the change of interfacial tension than the change of viscosity, the latter can be seen in Figure 6c. This indicates that the difference in the breakup time shown in Figure $6 \mathrm{~b}$ is more likely to be affected by the inherent nature of the interfacial tension. That is, for the higher interfacial tension, the drop has a stronger interfacial reforming capability. Therefore, once the drop deformation starts, the local surface of the drop (especially the terminals of the deformed drop) will be reshaped more quickly, making a shorter time for the deforming process. The influence of the dispersed phase viscosity on the breakup time is shown in Figure 6c. The dispersed phase viscosity can significantly hinder the drop deformation rate and also block the local drop surface reshaping. The former will directly prolong the breakup time of the drop, while the latter will induce a more significant deformation before drop breakup and thus increase the deforming time. It should benoted that during the deformation, the drop with high viscosity tends to stretch into a thin filament in the middle of the deformed drop, and may generate several small satellite droplets. What's more, all three images in Figure 6 show that the average breakup time monotonically increases with the increasing of the drop diameter. As the drop getting larger, the drop surface needs to undergo the deformation with a longer trajectory before the breakage. Considering that the transfer rate of energy at the drop surface will not change significantly with the increase of drop size, resulting in that the breakup time increases for the larger drop. 

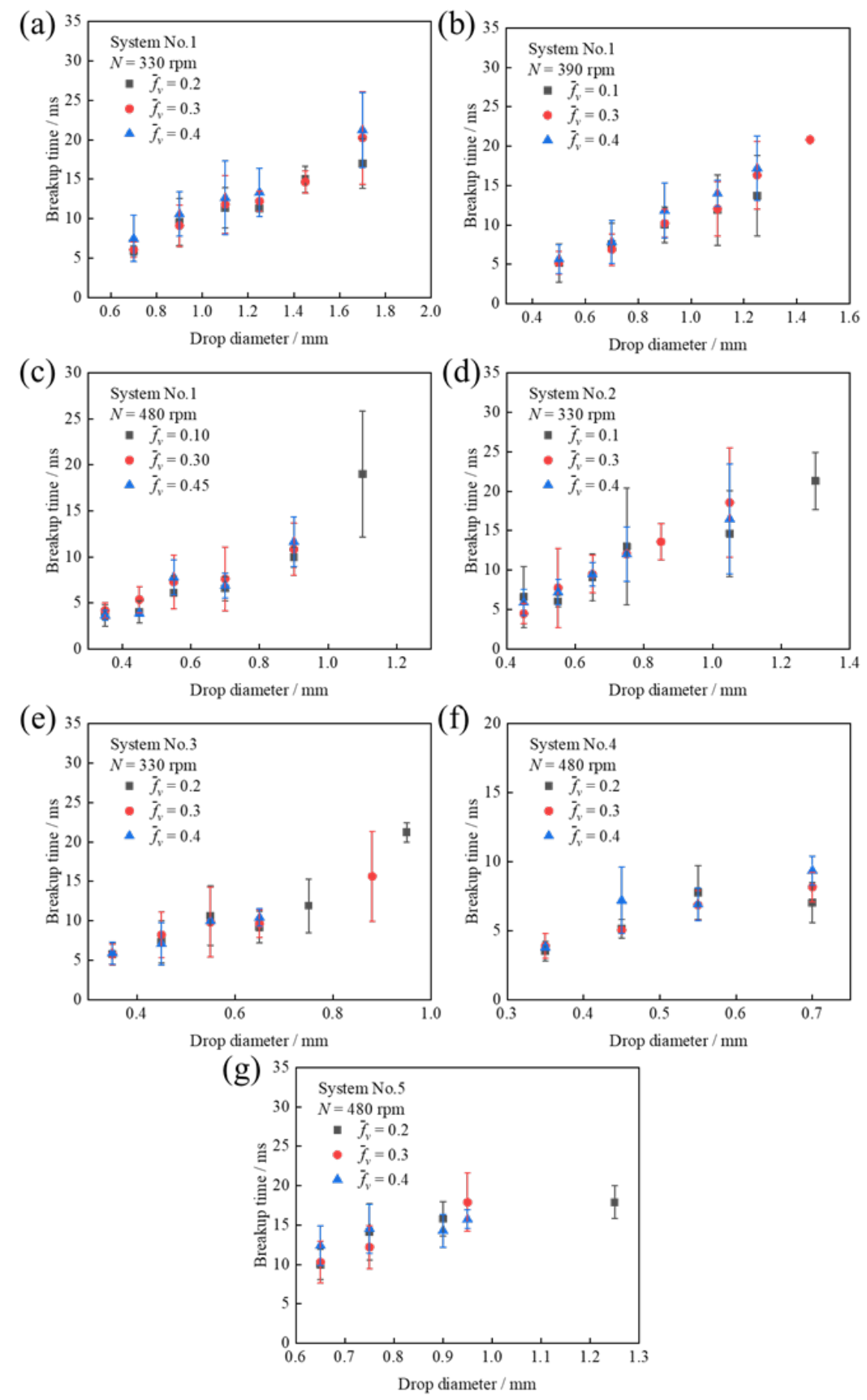

Figure 4 Influence of the volume fraction of the fragment on experimental breakup time. (a) System No.1, $N=330 \mathrm{rpm}$; (b) System No.1, $N=390 \mathrm{rpm}$; (c) System No.1, $N=480 \mathrm{rpm}$; (d) System No.2, $N=330 \mathrm{rpm}$; (e) System No.3, $N=330 \mathrm{rpm}$; (f) System No.4, $N=480$ rpm ; (g) System No.5, $N=480$ rpm. 

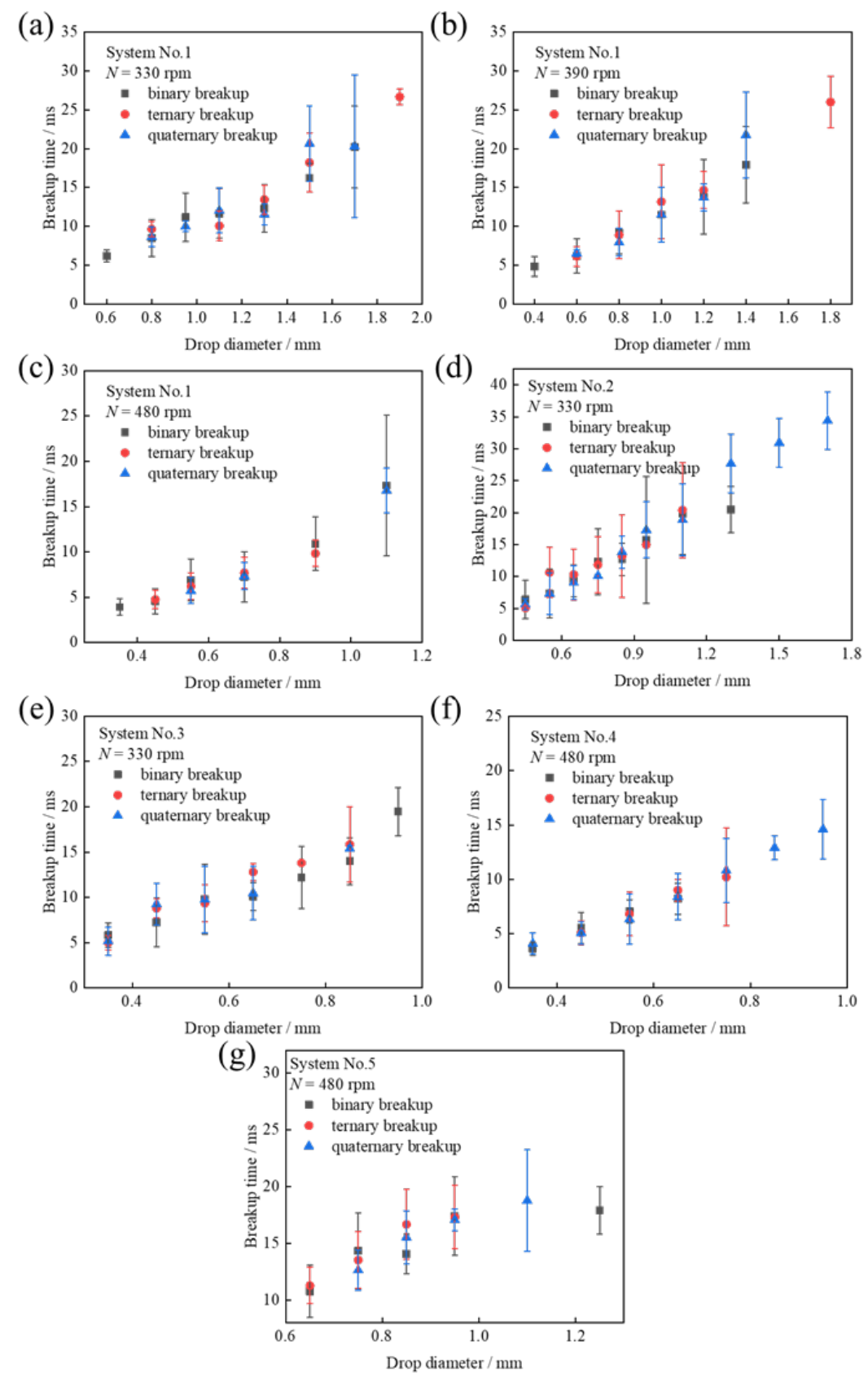

Figure 5 Influence of the number of the fragment on experimental breakup time. (a) System No.1, $N=330 \mathrm{rpm}$; (b) System No.1, $N=390 \mathrm{rpm}$; (c) System No.1, $N=480 \mathrm{rpm}$; (d) System No.2, $N=330 \mathrm{rpm}$; (e) System No.3, $N=330 \mathrm{rpm}$; (f) System No.4, $N=480 \mathrm{rpm}$ ; (g) System No.5, $N=480 \mathrm{rpm}$. 

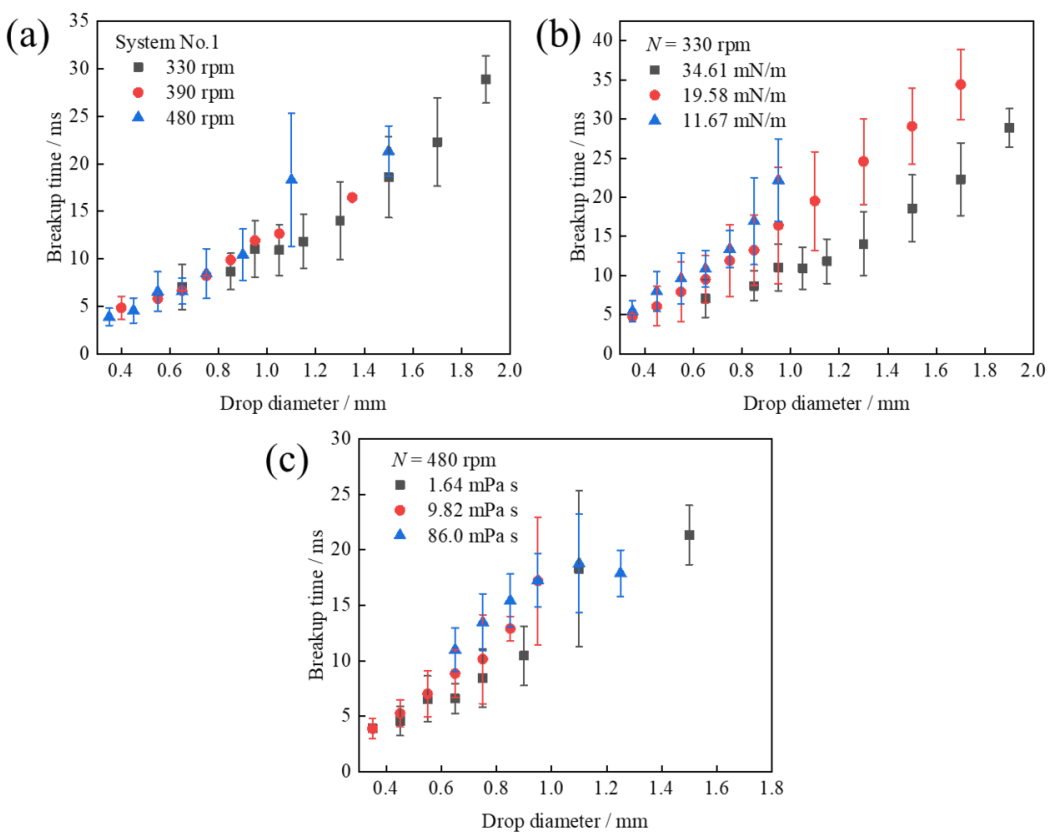

Figure 6 Experimental breakup time. (a) System No.1, $N=330 \sim 480$ rpm; (b) System No.1-3, $N=330 \mathrm{rpm}$; (c) System No.1,4-5, $N=480 \mathrm{rpm}$.

\section{Modeling analysis of the breakup time}

As mentioned above, the experimental studies on drop breakup time have been carried out by researchers with different systems. However, the quantitative description of the influences of operating parameters and

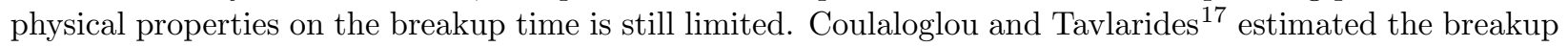
time using Equation 7:

Vankova et al. ${ }^{55}$ modified Equation 7 by introducing a dependency on the densities of two phases:

Where, is the Reynolds number in the drop.

Eastwood et al. ${ }^{16}$ investigated the influence of the drop viscosity on the deforming time and indicated that the breakup time is in silimar scale with the capillary time:

Maaß and Kraume ${ }^{30}$ proposed a new model for the breakage time in the turbulent regime:

Where is the classic rate of the elongation and is the capillary forces with the critical thread diameter of the elongated drop, . The limitation associated with Equation 10 is the lack of generality when applied to other equipment or systems. ${ }^{34}$

Based on the experimental results in the above section, the value of the breakup time depends on the drop size, interfacial tension, and the dispersed phase viscosity. That is to say, the intrinsic characteristics of the drop determine the value of breakup time. For a spherical drop, the natural frequency of then th-order shape oscillation represents its temporal properties. The fundamental mode of oscillation, corresponding to $n=2$, is the most important mode ${ }^{16,56,57}$. Thus, the oscillation period $T$ can be estimated according to the second-order surface oscillating frequency of drop, as is shown in Equation 11. 


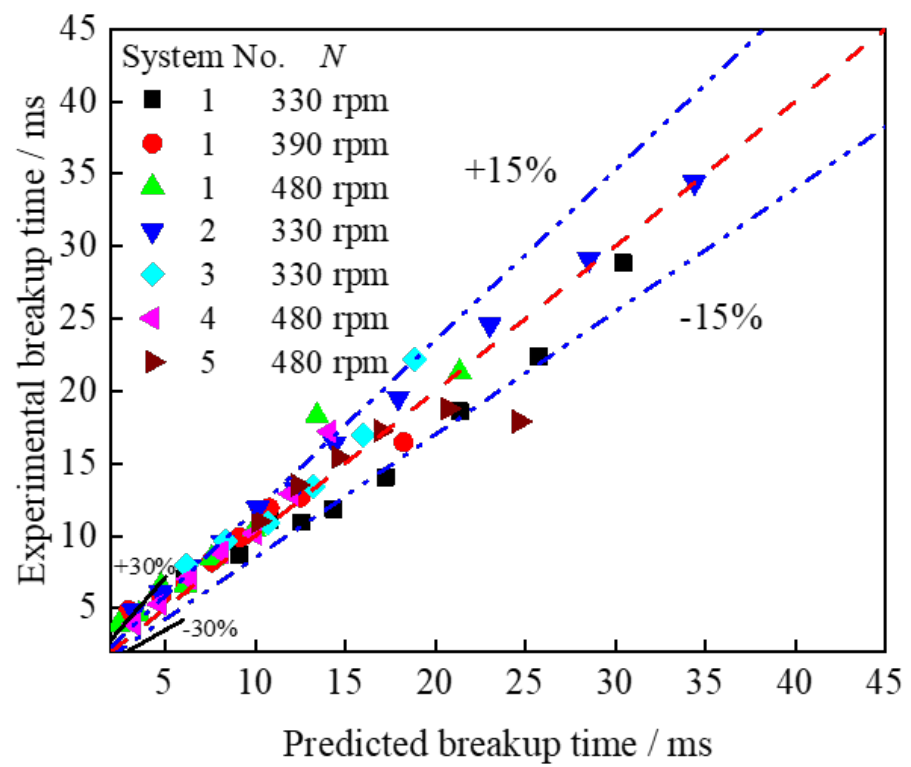

Figure 7 Experimental breakup time versus the predicted breakup time based on Equation 12.

Based on the analysis above, it can be reasonably assumed that the drop breakup time $t_{b}$ follows a linear relationship with the oscillation period. Moreover, the breakup time is slightly higher for drops with larger viscosity as shown in Figure 6c. For the high viscous drops, the influence of the viscosity on breakup time can be determined by introducing the Ohnesorge number, $O h=\mu_{\delta} /\left(\rho_{\delta} \sigma \delta\right)^{1 / 2}$. As a result, the empirical correlation 12 is proposed to predict the breakup time. The parameters in the correlation is determined based on least-squares fitting. The calculated results of the correlation were compared with the experimental data as shown in Figure 7 and a good agreement can be observed.

Solsvik and Jakobsen ${ }^{34}$ studied the drop breakup time by single drop experiments in a stirred liquid-liquid tank. They established polynomial/power functions for the breakup time of four kinds of drops (n-dodecane, toluene, petroleum, and 1-octanol), as presented in Equation 13. The drop diameter in their study varies from $0.5 \mathrm{~mm}$ to $4 \mathrm{~mm}$. In this study, Equation 13 was adopted to calculate the breakup times of the four kinds of oils and 8 points with equal intervals $(0.5 \mathrm{~mm})$ were selected, as shown in Figure 8 . These data points represent the average droplet breakup time in the experiments of Solsvik and Jakobsen ${ }^{34}$. Meanwhile, Equation 12 is used to calculate the breakup times for the droplets of the four kinds of oils and plotted in Figure 8. The results show that the model constructed in this study is in good agreement with the experimental data of Solsvik and Jakobsen ${ }^{34}$, which further verifies the accuracy and applicability of Equation 12. 


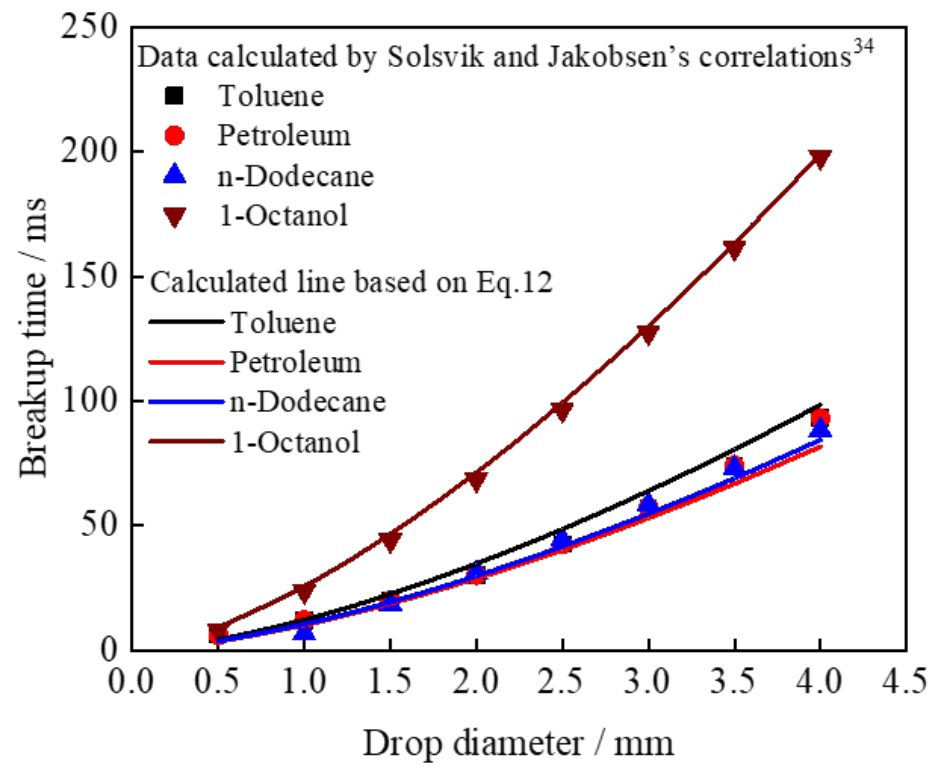

Figure 8 Comparison between the experimental breakup time of Solsvik and Jakobsen (2015) 34 and that calculated from Equation 12 in this study.

\section{Multiple breakage}

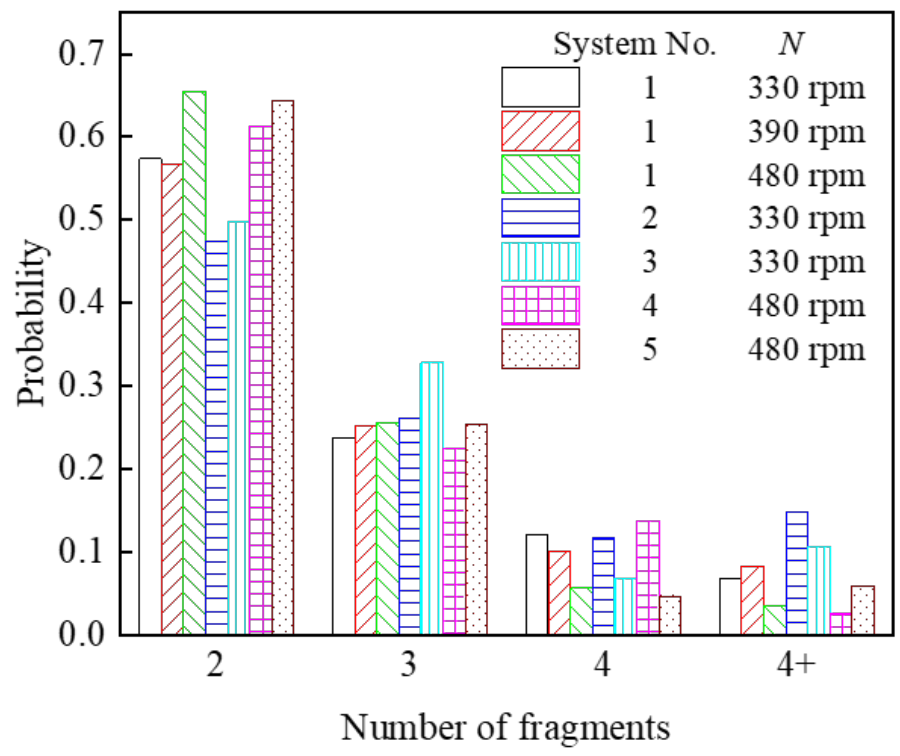

Figure 9 The proportion of the different numbers of fragments. 
In this section, the probability distribution of the number of fragments, the maximum stable drop diameter, and the percentage of binary breakage was discussed. Figure 9 shows the proportion of binary, ternary, quaternary, and quaternary+ breakage. Similar to our previous studies ${ }^{41,58}$, the binary breakup is dominant over the whole breakup events. Figure 9 also indicated that the occurrence probability of the multiple breakages is increasing for the drops with lower interfacial tension, which can be seen from the Systems No.2-3 in Figure 9. This is mainly due to the larger size of the drops relative to the maximum stable drop, which will be analyzed in detail in the following text.

The impeller Weber number ( $W e=\rho_{S} N^{2} \Delta^{3} / \sigma$ ) is widely used to model the Sauter mean diameter $\left(d_{32}\right)$ and the maximum stable drop diameter $(d$ max $) \cdot{ }^{59-63} \mathrm{~A}$ common conclusion with the vast majority of systems is that $d_{32}$ and $d$ max depend on the -0.6 power of $W e$ in stirred vessels. ${ }^{60,64,65}$ In such a scenario, we plotted the $d_{32}$ and $d_{\max }$ using the $W e^{-0.6}$ as the abscissa, as is shown in Figure 10a. It can be seen that except for the most viscous system (System No.5, $N=480 \mathrm{rpm}$ ), the $d_{32}$ and $d$ max display the linear dependence on the $W e^{-0.6}$. The least-squares fitted lines are thus plotted in Figure 10a. Considering the influence of the dispersed phase viscosity on the $d_{32}$ and $d_{\max }$, the mechanistic model proposed by Calabrese et al. ${ }^{66}$ and Wang and Calabrese ${ }^{67}$ can be adopted. The expression for the $d_{32}$ is shown in Equation 14. Moreover, Sprow ${ }^{68}$ proposed that the the maximum diameter is proportional to the average drop size, the conclusion is also valid in this study. Thus, the correlation for the $d$ maxwas expressed as Equation 15 . The fitting parameters for Equations 14, 15 were showed in Table 3.

And:

Where $D$ is the diameter of the impeller.

Table 3 Fitting parameters for Equations 14,15

\begin{tabular}{ll}
\hline Parameters & Value \\
\hline$c_{5}$ & 0.2928 \\
$c_{6}$ & 0.3500 \\
$c_{8}$ & 0.1568 \\
\hline
\end{tabular}

The comparison of the experimental data of the $d_{32}$ and $d_{\max }$ with the calculated results using Equations 14, 15 is shown in Figure 10b. It is indicated that the predicted results are in good agreement with the experimental data with an error of $\pm 10 \%$.
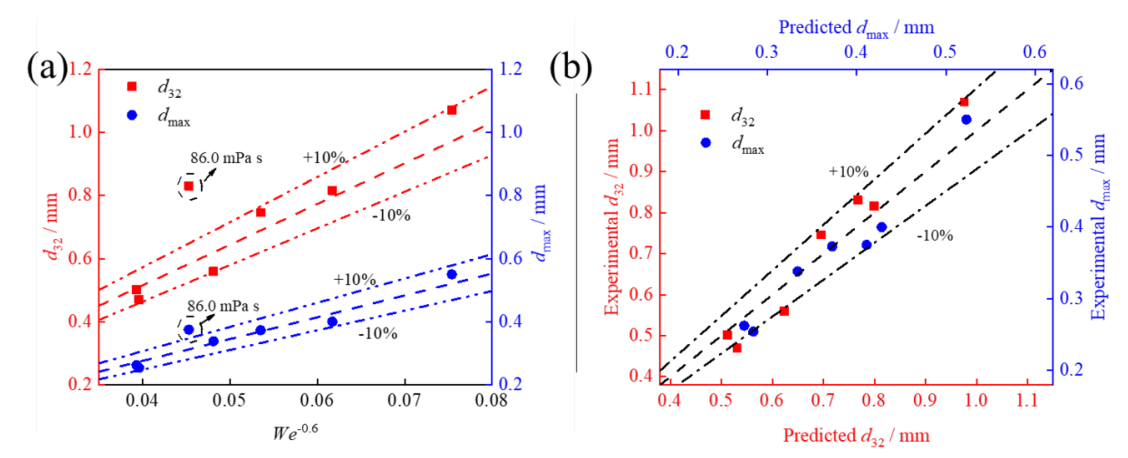

Figure 10 TheSauter mean diameter $\left(d_{32}\right)$ and the maximum stable drop diameter $\left(d_{\max }\right)$ in this study (a) versus $W e^{-0.6}$; (b) comparison between the experimental data with the predicted values.

Usually, the mechanism of drop deformation is deemed to be the consequence of eddy-drop interaction. A drop lied in a turbulent flow field undergoes the external disruptive stress and self-restoring stress. Whether a 
drop breaks up or not and how the drop deforms depend on the relative magnitudes of the two stresses. And the critical point determines the maximum stable diameter of the drop, $d$ max , the drop can break up only if its diameter is larger than $d_{\max }$. For the given system and operating conditions, the drop is more unstable when the drop size is further from the equilibrium size, and the drop is more likely to be deformed and broken. ${ }^{36}$ In other words, the multiple breakup characteristics of the drop are also more distinct. Generally, the percentage of binary breakup can be used to characterize the multiple drop breakup behaviors. Figure 11a presented the proportion of binary breakage for all systems in this study. It is indicated that the percentage of the binary breakup is lower for the larger drop, and varies with different systems and rotating speeds. According to the previous analysis, the influence of the above factors can be characterized by the relative distance to the $d_{\max }$. By defining a dimensionless parameter $\eta=d / d$ max , Figure 11a is transferred into Figure 11b. Meanwhile, we compared results in this study with the experimental results of Hao Zhou et al. ${ }^{44}$ in a pulsed disc and doughnut column. It can be seen from Figure $11 \mathrm{~b}$ that all data points lied within a narrow strip, which indicates that the defined parameter $\eta$ is appropriate to describe the relative stability of a drop.
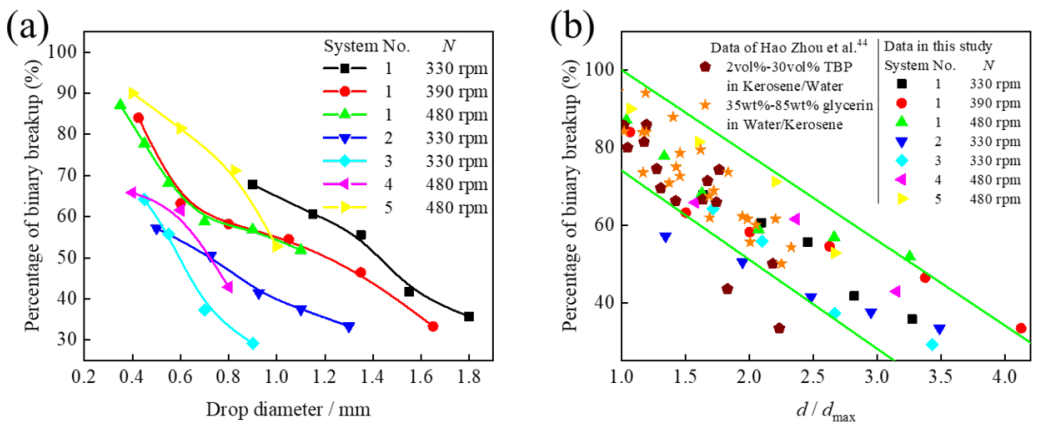

Figure 11 The percentage of the binary breakup. (a) versus drop diameter; (b) versus dimensionless diameter $\left(d / d_{\max }\right)$ and compare with the experimental result in a pulsed disc and doughnut column ${ }^{44}$.

\section{Breakup rate}

The drop breakup rate was determined experimentally based on Equation 1. Figure 12 shows the results of the drop breakup rate using the drop diameter as the abscissa. It can be seen from Figure 12a-c that breakup rate monotonously increases with increasing drop diameter. Moreover, Figure 12a,b indicated that the breakup rate gets larger for the larger rotating speed and lower interfacial tension. The reason is that increasing the rotating speed can strengthen the disruptive stress, as is shown in Equation 4,5, which leads to an increase in the drop breakup possibility. Besides, it is illustrated in Section 4.1 that the breakup time is almost independent of the rotating speed, making the breakup rate monotonously increases with the increase of rotating speeds. For the lower interfacial tension and larger drop diameter, the interfacial stress $\sigma_{\mathrm{I}}=6 \sigma / d$ of a drop is smaller, leading to the weaker ability to maintain drops without deforming. Thus, the breakup rate is larger for the smaller interfacial stress. Figure 12c shows the combined influences of interfacial tension and dispersed phase viscosity. As the interfacial tension is little different for the System No.1 and 5, the big distinction of the breakup rate is attributed to the different dispersed phase viscosity. 

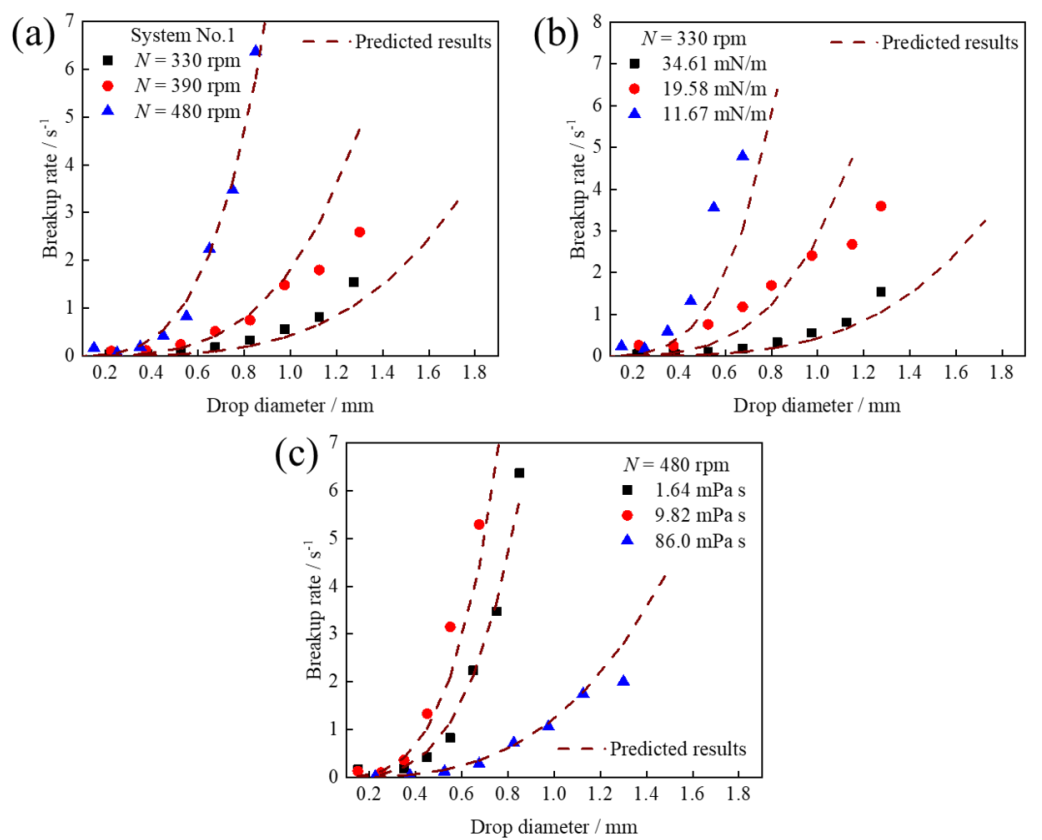

Figure 12 Comparison between the experimental breakup rate and predicted results using the breakup rate model of Han Zhou et al. $(2019){ }^{45}, S_{10}=11, \beta=2 . \quad(\alpha) \Sigma \psi \sigma \tau \varepsilon \mu$ No.1, $N=330 \sim 480 \rho \pi \mu \cdot(\beta) \Sigma \psi \sigma \tau \varepsilon \mu$ No.1-3, $N=330 \rho \pi \mu \cdot(\varsigma) \Sigma \psi \sigma \tau \varepsilon \mu$ No.1,4-5, $N=480 \rho \pi \mu$.

In our previous research ${ }^{45}$, an empirical correlation of the drop breakup rate was constructed based on the dimensionless analysis. The correlation is expressed as:

And:

Where $\sigma_{\text {drop }}$ is the drop restoring stress, representing the ability resisting the drop deformation. $\sigma_{\mathrm{v}, \mathrm{c}}$, and $\sigma_{\mathrm{v}, \mathrm{d}}$ represent the viscous stress of the continuous phase and the dispersed phase respectively. $\tau_{\mathrm{t}}$ is the disruptive stress, which can be calculated using Equation 5 in Section 3.2.

Equation 17 was adopted to predict the breakup rate in this study. The calculated results were plotted in Figure 12. It can be seen that a good agreement between the predicted value and the experimental breakup rate is obtained, which further proved the accuracy and expansibility of Equation 17. Moreover, it should also be pointed out that Equation 17 presents the monotone property of the breakup rate with increasing the drop diameter. Considering that the drop breakup time is getting larger with the increase of drop size, while the breakup probability of the drop has an upper limit of $100 \%$. Thus, the monotone property of Equation 17 can only be strictly valid when the breakup probability of the drop is relatively low. Based on the experimental breakup rate in Figure 12 and the correlation of the breakup time in Equation 12, the breakup possibility $\left(P_{\mathrm{b}}\right)$ in this study can be calculated using Equation 20. The results are then plotted in Figure 13. It can be seen that the values of $P_{\mathrm{b}}$ are all lower than $10 \%$, which further proved the applicability of the breakup model used in this study. 

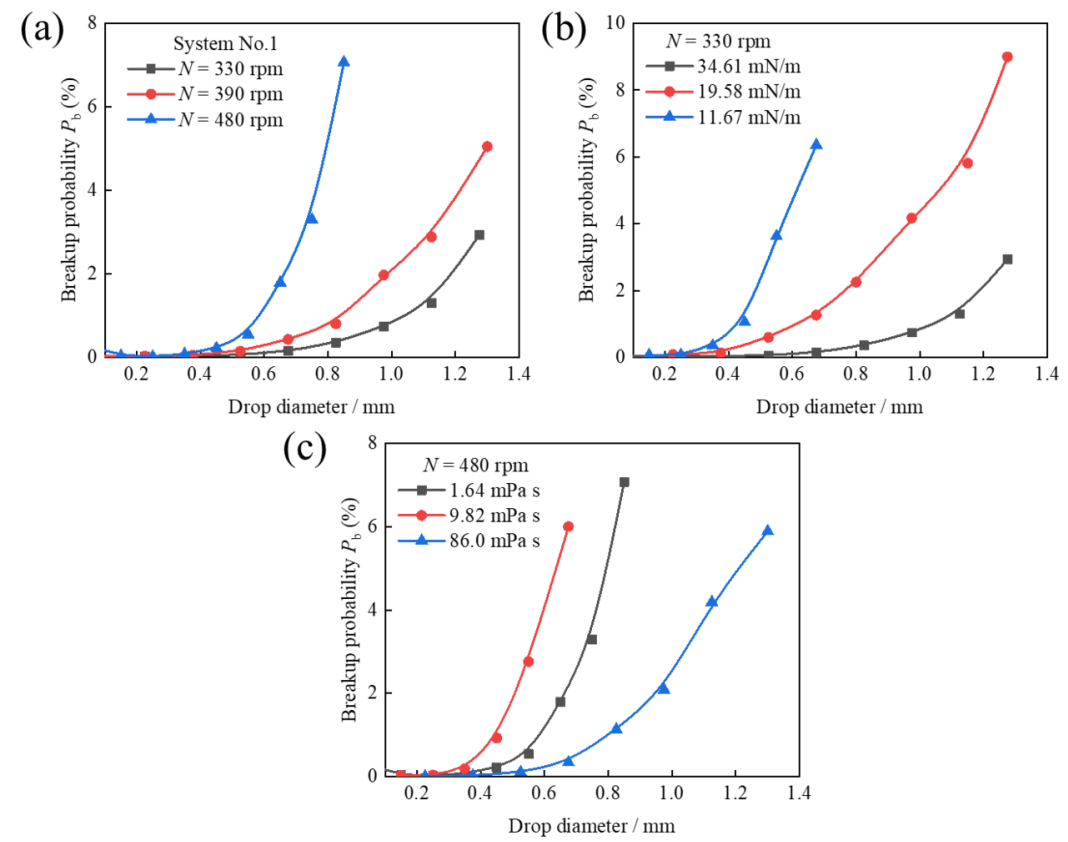

Figure 13 Drop breakup probability (a) System No.1, $N=330$ 480 rpm; (b) System No.1-3, $N=330 \mathrm{rpm}$; (c) System No.1,4-5, $N=480 \mathrm{rpm}$.

\section{Conclusion}

The drop breakup behavior was investigated through systematic experiments. The drop breakup time, breakup rate and the behavior of multiple breakage is discussed in this work. The definition of the breakup time is proposed as the time duration from the deformation of a spherical drop to the generation of the last fragment. The influences of the rotating speed, interfacial tension and the dispersed phase viscosity on the breakup time were analyzed. The experimental results indicated that the breakup time mainly depends on the interfacial tension and the drop diameter, slightly relies on the dispersed phased viscosity, while is almost independent of the rotating speed. An empirical correlation is proposed to predict the breakup time, and a good agreement was obtained between the predicted value and the experimental data in this study as well as in Solsvik and Jakobsen's work ${ }^{34}$.

The maximum stable drop diameter $d_{\max }$ is measured and shows a -0.6 power dependency of the impeller Weber number for the low viscous drop. For high viscous drop, the viscosity group is introduced to model the $d$ max. The percentage of the binary breakup is analyzed to investigate the behavior of the multiple breakage. It is shown that the percentage of the binary breakup depends on the dimensionless diameter $\eta$ $=d / d \max$.

Finally, the breakup rate was experimental measured. it has shown a good concordance of the predicted values with the experimental ones, which further verified the accuracy and extensibility of the breakup model proposed in our previous study.

\section{Acknowledgments}

We gratefully acknowledge the support of the National Natural Science Foundation of China (21776151, 21576147). 


\section{Notation}

$d$ diameter of the drop, $\mathrm{m}$

$d_{32}$ Sauter mean diameter, $\mathrm{m}$

$d_{r}$ the relative deviation

$d_{\max }$ maximum stable drop diameter, $\mathrm{m}$

$D$ diameter of the impeller, $\mathrm{m}$

$f_{v}$ volume ratio of the daughter droplet to the mother droplet

$k$ the turbulent kinetic energy, $\mathrm{m}^{2} / \mathrm{s}^{2}$

$n(d)$ number density function of the droplets, $\mathrm{m}^{-4}$

$N$ rotating speed of the impeller, $\mathrm{r} / \mathrm{s}$

Oh the Ohnesorge number

$P_{b}$ breakup probability

$t_{b}$ breakup time, ms

$t_{c}$ time duration, $\mathrm{s}$

$T$ the oscillation period of drop surface, $\mathrm{s}$

$V I$ viscosity group

We impeller Weber number

\section{Greek letter}

\begin{tabular}{ll}
\hline$\Gamma(d)$ & breakup rate function, $\mathrm{s}^{-1}$ \\
\hline$\epsilon$ & energy dissipation rate, $\mathrm{m}^{2} / \mathrm{s}^{3}$ \\
$\mu_{\mathrm{S}}$ & viscosity of the continuous phase, Pa s \\
$\mu_{\delta}$ & viscosity of the dispersed phase, Pa s \\
$?$ & viscosity ratio \\
& a distance parameter, $\mathrm{m}$ \\
$?$ & the dimensionless diameter \\
& Kolmogorov microscale, $\mathrm{m}$ \\
$\rho_{\mathrm{S}}$ & the distance over which the vortex velocity varies significantly, $\mathrm{m}$ \\
$\rho_{\delta}$ & continuous phase density, $\mathrm{kg} / \mathrm{m}^{3}$ \\
$\sigma$ & interfacial tension, $\mathrm{N} / \mathrm{m}$ \\
$\sigma_{\mathrm{I}}$ & interfacial stress, $\mathrm{N} / \mathrm{m}^{2}$ \\
$\sigma_{\mathrm{v}, \mathrm{c}}$ & deforming viscous stress, $\mathrm{N} / \mathrm{m}^{2}$ \\
$\sigma_{\mathrm{v}, \mathrm{d}}$ & Internal viscous stress, $\mathrm{N} / \mathrm{m}^{2}$ \\
$\sigma_{\mathrm{drop}}$ & drop restoring stress, $\mathrm{N} / \mathrm{m}^{2}$ \\
$?_{t}$ & the disruptive stress, $\mathrm{N} / \mathrm{m}^{2}$ \\
\hline &
\end{tabular}

\section{Abbreviations}

DSD drop size distribution

DDSD daughter drop size distribution 
RT The Rushton Turbine

\section{Reference}

1. Kislik VS. Solvent Extraction : Classical and Novel Approaches . Elsevier Science; 2011.

2. Liu J-Q, Li S-W, Jing S. Axial Mixing and Mass Transfer Performance of an Annular Pulsed Disc-andDoughnut Column. Solvent Extr Ion Exch . 2015;33(6):592-606.

3. Marchisio DL, Fox RO. Multiphase Reacting Flows: Modelling and Simulation. Wien: Springer-Verlag; 2007.

4. Thickett SC, Gilbert RG. Emulsion polymerization: State of the art in kinetics and mechanisms. Polymer . 2007;48(24):6965-6991.

5. Windhab EJ, Dressler M, Feigl K, Fischer P, Megias-Alguacil D. Emulsion processing - from single-drop deformation to design of complex processes and products. Chem Eng Sci . 2005;60(8):2101-2113.

6. Vankova N, Tcholakova S, Denkov ND, Ivanov IB, Vulchev VD, Danner T. Emulsification in turbulent flow: 1. Mean and maximum drop diameters in inertial and viscous regimes. $J$ Colloid Interface Sci . 2007;312(2):363-380.

7. Ramkrishna D. Population Balances: Theory and Applications to Particulate Systems in Engineering . Elsevier; 2000.

8. Coulaloglou CA, Tavlarides LL. Drop size distributions and coalescence frequencies of liquid-liquid dispersions in flow vessels. AIChE $J$. 1976;22(2):289-297.

9. Bart H-J, Jildeh H, Attarakih M. Population Balances for Extraction Column Simulations-An Overview. Solvent Extr Ion Exch . 2020;38(1):14-65.

10. Kopriwa N, Buchbender F, Ayesterán J, Kalem M, Pfennig A. A Critical Review of the Application of Drop-Population Balances for the Design of Solvent Extraction Columns: I. Concept of Solving DropPopulation Balances and Modelling Breakage and Coalescence. Solvent Extr Ion Exch . 2012;30(7):683-723.

11. Falzone S, Buffo A, Vanni M, Marchisio DL. Simulation of Turbulent Coalescence and Breakage of Bubbles and Droplets in the Presence of Surfactants, Salts, and Contaminants. In: Parente A, De Wilde J, eds.Advances in Chemical Engineering. Vol 52. Academic Press; 2018:125-188.

12. Sathyagal AN, Ramkrishna D, Narsimhan G. Droplet breakage in stirred dispersions. Breakage functions from experimental drop-size distributions. Chem Eng Sci . 1996;51(9):1377-1391.

13. Kostoglou M, Karabelas AJ. A contribution towards predicting the evolution of droplet size distribution in flowing dilute liquid/liquid dispersions. Chem Eng Sci . 2001;56(14):4283-4292.

14. Kostoglou M, Karabelas AJ. Toward a unified framework for the derivation of breakage functions based on the statistical theory of turbulence. Chem Eng Sci . 2005;60(23):6584-6595.

15. O'Rourke AM, MacLoughlin PF. A study of drop breakage in lean dispersions using the inverse-problem method. Chem Eng Sci . 2010;65(11):3681-3694.

16. Eastwood CD, Armi L, Lasheras JC. The breakup of immiscible fluids in turbulent flows. J Fluid Mech . 2004;502:309-333.

17. Coulaloglou CA, Tavlarides LL. Description of interaction processes in agitated liquid-liquid dispersions. Chem Eng Sci . 1977;32(11):1289-1297.

18. Narsimhan G, Gupta JP, Ramkrishna D. A model for transitional breakage probability of droplets in agitated lean liquid-liquid dispersions. Chem Eng Sci . 1979;34(2):257-265. 
19. Hsia MA, Tavlarides LL. Simulation analysis of drop breakage, coalescence and micromixing in liquidliquid stirred tanks. Chem Eng J . 1983;26(3):189-199.

20. Narsimhan G, Nejfelt G, Ramkrishna D. Breakage functions for droplets in agitated liquid-liquid dispersions. AIChE J . 1984;30(3):457-467.

21. Chatzi EG, Erickson AD, Kiparissides C. Generalized model for prediction of the steady-state drop size distributions in batch stirred vessels. Ind Eng Chem Res . 1989;28(11):1704-1711.

22. Tsouris C, Tavlarides LL. Breakage and coalescence models for drops in turbulent dispersions. AIChE $J$ . 1994;40(3):395-406.

23. Luo H, Svendsen HF. Theoretical model for drop and bubble breakup in turbulent dispersions. AIChE J . 1996;42(5):1225-1233.

24. Bahmanyar H, Dean DR, Dowling IC, Ramlochan KM, Slater MJ, Yu W. Studies of drop break-up in liquid-liquid systems in a rotating disc contactor. Part II: Effects of mass transfer and scale-up. Chem Eng Technol . 1991;14(3):178-185.

25. Bahmanyar H, Slater MJ. Studies of drop break-up in liquid-liquid systems in a rotating disc contactor. Part I: Conditions of no mass transfer. Chem Eng Technol . 1991;14(2):79-89.

26. Cabassud M, Gourdon C, Casamatta G. Single drop break-up in a Kühni column. Chem Eng $J$. 1990;44(1):27-41.

27. Fang J, Godfrey JC, Mao Z-Q, Slater MJ, Gourdon C. Single liquid drop breakage probabilities and characteristic velocities in Kühni columns. Chem Eng Technol . 1995;18(1):41-48.

28. Galinat S, Masbernat O, Guiraud P, Dalmazzone C, Nor”k C. Drop break-up in turbulent pipe flow downstream of a restriction. Chem Eng Sci . 2005;60(23):6511-6528.

29. Jareš J, Procházka J. Break-up of droplets in Karr reciprocating plate extraction column. Chem Eng Sci . 1987;42(2):283-292.

30. Maaß S, Kraume M. Determination of breakage rates using single drop experiments. Chem Eng Sci . 2012;70:146-164.

31. Percy JS, Sleicher CA. Drop breakup in the flow of immiscible liquids through an orifice in a pipe. AIChE J . 1983;29(1):161-164.

32. Gourdon C, Casamatta G, Angelino H. Single drop experiments with liquid test systems: A way of comparing two types of mechanically agitated extraction columns. Chem Eng J . 1991;46(3):137-148.

33. Galinat S, Risso F, Masbernat O, Guiraud P. Dynamics of drop breakup in inhomogeneous turbulence at various volume fractions. J Fluid Mech . 2007;578:85-94.

34. Solsvik J, Jakobsen HA. Single drop breakup experiments in stirred liquid-liquid tank. Chem Eng Sci . 2015;131:219-234.

35. Korb C, Bart H-J. Solvent extraction in columns in a droplet breakage domain. Hydrometallurgy . 2017;173:71-79.

36. Ashar M, Arlov D, Carlsson F, Innings F, Andersson R. Single droplet breakup in a rotor-stator mixer. Chem Eng Sci . 2018;181:186-198.

37. Solsvik J, Maaß S, Jakobsen HA. Definition of the Single Drop Breakup Event. Ind Eng Chem Res . $2016 ; 55(10): 2872-2882$.

38. Liu H, Jing S, Fang Q, Li S. Droplet Breakup in a Square-Sectioned Pulsed Disc and Doughnut Column. Ind Eng Chem Res . 2016;55(7):2242-2251. 
39. Fang Q, Jing D, Zhou H, Li S. Population balance of droplets in a pulsed disc and doughnut column with wettable internals. Chem Eng Sci . 2017;161:274-287.

40. Zhou H, Jing S, Fang Q, Li S, Lan W. Direct measurement of droplet breakage in a pulsed disc and doughnut column. AIChE J . 2017;63(9):4188-4200.

41. Zhou H, Yu X, Jing S, Zhou H, Lan W, Li S. Measurement of droplet breakage in a pump-mixer. Chem Eng Sci . 2019;195:23-38.

42. Hesketh RP, Etchells AW, Russell TWF. Experimental observations of bubble breakage in turbulent flow. Ind Eng Chem Res . 1991;30(5):835-841.

43. Andersson R, Andersson B. On the breakup of fluid particles in turbulent flows. AIChE J . 2006;52(6):20202030 .

44. Zhou H, Jing S, Yu X, Zhou H, Lan W, Li S. Study of droplet breakage in a pulsed disc and doughnut column-Part I: Experiments and correlations. Chem Eng Sci . 2019;197:172-183.

45. Zhou H, Yang J, Jing S, Lan W, Zheng Q, Li S. Influence of Dispersed-Phase Viscosity on Droplet Breakup in a Continuous Pump-Mixer.Ind Eng Chem Res . 2019;58(51):23458-23467.

46. Zhang Y, Gao Z, Li Z, Derksen JJ. Transitional flow in a Rushton turbine stirred tank. AIChE J . 2017;63(8):3610-3623.

47. Placek J, Tavlarides LL. Turbulent flow in stirred tanks. Part I: Turbulent flow in the turbine impeller region. AIChE J . 1985;31(7):1113-1120.

48. Placek J, Tavlarides LL, Smith GW, Fořt I. Turbulent flow in stirred tanks. Part II: A two-scale model of turbulence. AIChE J . 1986;32(11):1771-1786.

49. Han L, Gong S, Li Y, et al. Influence of energy spectrum distribution on drop breakage in turbulent flows. Chem Eng Sci . 2014;117:55-70.

50. Bates RL, Fondy PL, Corpstein RR. Examination of Some Geometric Parameters of Impeller Power. Ind Eng Chem Process Des Dev . 1963;2(4):310-314.

51. Shinnar R. On the behaviour of liquid dispersions in mixing vessels. J Fluid Mech . 1961;10(2):259-275.

52. Batchelor GK. The Theory of Homogeneous Turbulence . Cambridge University Press.; 1956.

53. Kolmogorov A. On the breakage of drops in a turbulent flow. In: Vol 66. ; 1949:825-828.

54. Karimi M, Andersson R. Stochastic simulation of droplet breakup in turbulence. Chem Eng $J$. 2020;380:122502.

55. Vankova N, Tcholakova S, Denkov ND, Vulchev VD, Danner T. Emulsification in turbulent flow: 2. Breakage rate constants. J Colloid Interface Sci . 2007;313(2):612-629.

56. Lamb H. Hydrodynamics . Cambridge university press; 1993.

57. Sevik M, Park SH. The Splitting of Drops and Bubbles by Turbulent Fluid Flow. J Fluids Eng . 1973;95(1):53-60.

58. Zhou H, Yang J, Jing S, Lan W, Zheng Q, Li S. Influence of Dispersed-Phase Viscosity on Droplet Breakup in a Continuous Pump-Mixer.Ind Eng Chem Res . November 2019.

59. Desnoyer C, Masbernat O, Gourdon C. Experimental study of drop size distributions at high phase ratio in liquid-liquid dispersions. Chem Eng Sci . 2003;58(7):1353-1363.

60. Singh KK, Mahajani SM, Shenoy KT, Ghosh SK. Representative drop sizes and drop size distributions in A/O dispersions in continuous flow stirred tank. Hydrometallurgy . 2008;90(2):121-136. 
61. Maaß S, Metz F, Rehm T, Kraume M. Prediction of drop sizes for liquid-liquid systems in stirred slim reactors-Part I: Single stage impellers. Chem Eng J . 2010;162(2):792-801.

62. Razzaghi K, Shahraki F. On the effect of phase fraction on drop size distribution of liquid-liquid dispersions in agitated vessels. Chem Eng Res Des . 2010;88(7):803-808.

63. Maaß S, Paul N, Kraume M. Influence of the dispersed phase fraction on experimental and predicted drop size distributions in breakage dominated stirred systems. Chem Eng Sci . 2012;76:140-153.

64. Abidin MIIZ, Raman AAA, Nor MIM. Mean drop size correlations and population balance models for liquid-liquid dispersion. AIChE J . 2015;61(4):1129-1145.

65. Parvizi S, Alamdari EK, Hashemabadi SH, Kavousi M, Sattari A. Investigating Factors Affecting on the Efficiency of Dynamic Mixers. Miner Process Extr Metall Rev . 2016;37(5):342-368.

66. Calabrese RV, Wang CY, Bryner NP. Drop breakup in turbulent stirred-tank contactors. Part III: Correlations for mean size and drop size distribution. AIChE J . 1986;32(4):677-681.

67. Wang CY, Calabrese RV. Drop breakup in turbulent stirred-tank contactors. Part II: Relative influence of viscosity and interfacial tension. AIChE J . 1986;32(4):667-676.

68. Sprow FB. Distribution of drop sizes produced in turbulent liquid-liquid dispersion. Chem Eng Sci . 1967;22(3):435-442. 